\title{
New 3-D Combined Inversion Scheme Using Response Functions Free From Galvanic Distortion
}

\author{
Yoshiya Usui ( $\nabla$ yusui@eri.u-tokyo.ac.jp ) \\ University of Tokyo: Tokyo Daigaku https://orcid.org/0000-0001-5753-6568 \\ Makoto Uyeshima \\ The University of Tokyo: Tokyo Daigaku
}

\section{Research Article}

Keywords: magnetotelluric inversion, Network-MT method, galvanic distortion, finite element method, phase tensor, magnetic transfer function, tetrahedral mesh

Posted Date: August 23rd, 2021

DOI: https://doi.org/10.21203/rs.3.rs-798901/v1

License: (1) This work is licensed under a Creative Commons Attribution 4.0 International License. Read Full License 
$1 \quad$ New 3-D Combined Inversion Scheme Using Response Functions Free from

\section{Galvanic Distortion}

3 Author \#1: Yoshiya Usui, Earthquake Research Institute, the University of Tokyo, 1-1-1,

4 Yayoi, Bunkyo, Tokyo, 113-0032 Japan., yusui@eri.u-tokyo.ac.jp

5 Author \#2: Makoto Uyeshima, Earthquake Research Institute, the University of Tokyo,

6 1-1-1, Yayoi, Bunkyo, Tokyo, 113-0032 Japan., uyeshima@eri.u-tokyo.ac.jp

$7 \quad$ Corresponding author: Yoshiya Usui (yusui@eri.u-tokyo.ac.jp) 


\section{Abstract}

10 The combined inversion using distortion-free response functions is an effective

11 approach to robustly estimate the 3-D electrical resistivity structure against the

12 distortions caused by near-surface resistivity anomalies. However, previous combined

13 inversion analyses have presented a significant dependency of the inversion results on

14 initial and prior models. Therefore, in this study, we evaluated the effectiveness of the

15 following two new types of 3-D combined inversion using distortion-free response

16 functions: one uses the phase tensor and the vertical and inter-station horizontal

17 magnetic transfer functions, while the other uses the Network-MT response functions,

18 in addition to the former. Because long dipoles are used, the Network-MT response

19 function is negligibly affected by galvanic distortion. To access the combined inversion

20 approach, we developed a novel 3-D inversion scheme combining the response

21 functions of the usual magnetotelluric measurements and the Network-MT response

22 function. The synthetic inversion analysis demonstrated that both of the proposed

23 combined inversions can recover the characteristic resistivity distributions of the target

24 model without a significant dependence on the initial models, at least in the shallow 
25 part. These results demonstrate that the combined inversions using only distortion-free

26 response functions have the potential to estimate subsurface resistivity more robustly

27 than what was previously thought. Furthermore, we confirmed that the combined

28 inversion using the Network-MT response function can make the resultant resistivity

29 structure closer to the actual one and enhance the stability of the inversion. This result

30 suggests that the combined use of the Network-MT response function is the preferred

31 approach.

32

\section{Keywords}

34 magnetotelluric inversion, Network-MT method, galvanic distortion, finite element

35 method, phase tensor, magnetic transfer function, tetrahedral mesh

37 1. Introduction

38 The three-dimensional inversion of magnetotelluric (MT) data has become common in

39 recent decades. This is because of the increase in computational resources and the

40 widespread use of practical 3-D MT inversion codes, such as WSINV3DMT 
41 (Siripunvaraporn et al. 2005; Siripunvaraporn and Egbert 2009) and ModEM (Egbert

42 and Kelbert 2012; Kelbert et al. 2014). However, the galvanic distortion of the MT

43 impedance tensor has long been recognized as a major problem in estimating subsurface

44 electrical resistivity structures. Galvanic distortion, which is caused by lateral small-

45 scale inhomogeneities near the Earth's surface, distorts the electrical field and, hence,

46 the estimated impedance tensor (e.g., Ogawa 2002; Simpson and Bahr 2005).

48 One effective approach to robustly estimate the subsurface electrical resistivity structure

49 against the galvanic distortion is to use the response functions free from the galvanic

50 distortion as datasets in the MT inversion (e.g., Patro et al. 2013; Tietze et al. 2015;

51 Campanyà et al. 2016). Patro et al. (2013) developed a 3-D inversion scheme using the

52 phase tensor (PT) (Caldwell et al. 2004) as the input data. They demonstrated that the

53 PT inversion can provide the proper electrical resistivity structure by performing

54 synthetic inversion tests. Later, Tietze et al. (2015) developed a 3-D combined inversion

55 using PT and the vertical magnetic transfer function (VMTF) and demonstrated that the

56 integration of VMTF improves the recovery of the actual resistivity structure. In 
57 addition, Campanyà et al. (2016) assessed the sensitivity of the impedance tensor,

58 VMTF, and the inter-station horizontal magnetic transfer function (HMTF) to

59 subsurface resistivity anomalies by performing synthetic inversions with different

60 combinations of response functions. Campanyà et al. (2016) demonstrated that the

61 combined use of VMTF and HMTF with the impedance tensor recovers the resistivity

62 structure more accurately than using the impedance tensor alone. However, no study has

63 yet examined the combined inversion of PT, VMTF, and HMTF.

64

65 The previous studies using distortion-free response functions presented a deficiency in

66 robustness. Patro et al. (2013) and Tietze et al. (2015) showed that the resultant

67 electrical resistivity structure of the inversion using either or both PT and VMTF

68 depends significantly on the initial and prior models. They concluded that a sensible

69 selection of the prior and initial models is essential for recovering a reliable resistivity

70 structure. Campanyà et al. (2016) demonstrated that the inversion only using VMTF and

71 HMTF failed to recover the subsurface resistivity variation in the vertical direction and

72 the absolute resistivity values. They suggested that the inversion using either VMTF or 
73 HMTF is reliable only when additional information defining the background resistivity

74 structure is provided. As discussed in the previous studies, the dependency on the prior

75 and initial models should be caused by the low sensitivity of PT, VMTF, and HMTF to

76 the absolute values of the subsurface electrical resistivity.

78 One possible technique to overcome the problem by adding information regarding the

79 absolute values of the subsurface resistivity structure for inversion is the use of the

80 response function of the Network-MT (NMT) measurement (Uyeshima 2007), where

81 metallic telephone line networks are used to measure the electrical potential difference

82 between two distant electrodes (Uyeshima 2007). Because dipoles longer than several

83 kilometers are used in the NMT measurement, the response functions of the NMT

84 measurement (NMTRF) are negligibly affected by the galvanic distortion (Uyeshima et

85 al. 2001; Uyeshima 2007). In addition, NMTRF is significantly sensitive to the absolute

86 values of the subsurface resistivity structure, like the impedance tensor. Thus, it is

87 expected that the combined inversion with NMTRF allows the estimation of the

88 electrical resistivity structure with the correct scale. Recently, Usui et al. (2021) 
89 developed a 2-D combined inversion method using the usual MT response functions

90 (apparent resistivity and phase) and NMTRF. Usui et al. (2021) confirmed that the

91 combined inversion provides the correct resistivity structure despite the response

92 functions of the usual MT stations being significantly affected by galvanic distortion.

94 In addition, the combined use of NMTRF is advantageous because it enhances the

95 sensitivity to deep structures. Long dipole measurements of the NMT method make the

96 signal-to-noise ratio of the observed electrical field higher than that of the standard MT

97 measurement. In addition, well-maintained telephone lines enable us to perform long-

98 term observations from several months to several years (Kinoshita et al. 1989;

99 Uyeshima et al. 1989), allowing us to increase the signal-to-noise ratio by stacking the

100 data. Consequently, we can estimate NMTRF with small errors, including periods

101 longer than several thousand seconds, which assists in estimating a reliable electrical

102 resistivity down to the upper mantle. Siripunvaraporn et al. (2004) developed a 3-D

103 inversion scheme using NMTRF. However, no one has proposed a 3-D inversion

104 scheme that combines the response functions of the standard MT measurements and 
NMTRF.

106

107 In this study, we assessed the effectiveness of two new combined 3-D inversions using

108 response functions free from galvanic distortion, one of which is the combined

109 inversion of the PT, VMTF, and HMTF. All the response functions can be estimated

110 from the electromagnetic field observed by standard MT measurements. The second

111 inversion combines the PT, VMTF, HMTF, and NMTRF. To assess the second

112 combined inversion, we developed a novel 3-D inversion scheme that uses both

113 NMTRF and the response function of the standard MT measurements because no such

114 3-D inversion scheme exists. We performed synthetic inversions utilizing the same

115 model used in a previous study (Tietze et al. 2015) and compared the inversion results.

116 In the following sections, we first present the algorithm of the combined inversion

117 scheme used in this study, including the treatments of NMTRF in the inversion. Next,

118 we describe the synthetic inversion tests to evaluate the performance of the combined

119 inversion approaches and discuss the results of the combined inversions. 


\section{Algorithm of the combined inversion scheme}

122 We used the inversion scheme based on the 3-D MT inversion code of Usui (2015) and

123 Usui et al. (2017). This section summarizes the basic inversion algorithm and the

124 treatments of NMTRF in the forward calculation and inversion. The finite element

125 method with a tetrahedral mesh is used to obtain the electromagnetic field of the

126 computational domain. The governing equation is the vector Helmholtz equation for the

127 electric field with $\mathrm{e}^{-\mathrm{i} \omega \mathrm{t}}$ time dependence.

$$
\nabla \times \nabla \times \mathbf{E}=\mathrm{i} \omega \mu_{0} \sigma \mathbf{E}
$$

$128 \operatorname{In}(1), \mathbf{E} \in \mathbb{C}^{3}$ denotes the electric field, $\omega$ is the angular frequency, $\mu_{0}$ is the

129 magnetic permeability of free space, and $\sigma$ is the electrical conductivity. The

130 computational domain $\Omega$ indicates a rectangular geometry, and all the boundary planes

$131 \partial \Omega$ of $\Omega$ are perpendicular to the orthogonal coordinate axes. The Dirichlet boundary

132 condition is set on the entire boundary, $\partial \Omega$. On the top of $\Omega$, a uniform source electric

133 field is specified, while the tangential electric field is designed to be zero at the bottom

134 of $\Omega$, which corresponds to the condition that the perfect conductor locates below the

135 bottom. The electric field calculated by the 2-D forward calculation is provided on the 
136 two sides parallel to the source field. The 2-D forward problems (TM mode) are solved

137 by the Galerkin FEM using the triangular edge-based element with the lowest order (Jin

138 2002). On the other hand, the tangential electric field is forced to be zero on the sides

139 perpendicular to the source field. The boundary value problem is solved by the Galerkin

140 FEM (Jin 2002) using the edge-based tetrahedral element. Within each element, the

141 electric field can be approximated as follows:

$$
\mathbf{E}^{e}=\sum_{\mathrm{k}=1}^{6} \mathbf{N}^{e} \mathrm{E}^{e}{ }_{i}
$$

142 where $\mathbf{N}^{e}{ }_{i} \in \mathbb{R}^{3}$ and $\mathrm{E}^{e}{ }_{i} \in \mathbb{C}$ denote the vector basis function (Jin 2002) and the

143 tangential component of the electric field on the $i$-th edge of the element, respectively.

144 As shown in Usui (2015), we can obtain a linear equation for each element by using the

145 Galerkin method as follows:

$$
\mathbf{K}^{\mathrm{e}} \mathbf{x}^{e}=\mathbf{b}^{e}
$$

146 where $\mathbf{x}^{e}=\left(\mathrm{E}^{e}{ }_{1}, \ldots, \mathrm{E}^{e}{ }_{6}\right)^{T} \in \mathbb{C}^{6}$ is the solution vector, $\mathbf{b}^{e} \in \mathbb{C}^{6}$ denotes the right-

147 hand-side vector including the boundary condition, and the $i$-th row and $j$-th column of

148 the coefficient matrix $\mathbf{K}^{e} \in \mathbb{C}^{6 \times 6}$ is expressed as follows:

$$
\iiint_{\mathrm{V}^{e}}\left[\left(\nabla \times \mathbf{N}_{i}^{e}\right) \cdot\left(\nabla \times \mathbf{N}_{j}^{e}\right)-\mathrm{i} \omega \mu_{0} \sigma \mathbf{N}^{e}{ }_{i} \cdot \mathbf{N}^{e}{ }_{j}\right] \mathrm{dV}
$$


149 In Equation (4), $\mathrm{V}^{e}$ denotes the volume occupied by the $e$-th element. By assembling

150 all the element equations, we can obtain the following linear equation.

$$
\mathbf{K} \mathbf{x}=\mathbf{b}
$$

151 The dimension of (5) is the number of unknown tangential components of the electric

152 field at the element edges, and the coefficient matrix $\mathbf{K}$ is a symmetrical sparse

153 complex matrix. MKL PARDISO is used to solve the linear equation. To calculate the

154 response functions as indicated below, forward computation is performed twice for each

155 frequency by appending a source field in the $\mathrm{x}$-direction $\left(\mathrm{E}_{\mathrm{x}}\right.$-polarization $)$ and $\mathrm{y}$ -

156 direction ( $\mathrm{E}_{\mathrm{y}}$-polarization). After obtaining the electric field by solving the linear

157 equation above, the magnetic field $\mathbf{H}$ is calculated from the electric field using

158 Faraday's law.

$$
\nabla \times \mathbf{E}=\mathrm{i} \omega \mu_{0} \mathbf{H}
$$

159 From the results of the $\mathrm{E}_{\mathrm{x}}$-polarization and $\mathrm{E}_{\mathrm{y}}$-polarization, we can calculate the

160 frequency response function at each observation station. VMTF $\mathbf{T}_{v} \in \mathbb{C}^{2}$ is calculated

161 as follows: 


$$
\mathbf{T}_{v}=\left(\begin{array}{c}
\mathrm{T}_{\mathrm{zx}} \\
\mathrm{T}_{\mathrm{zy}}
\end{array}\right)=\left(\begin{array}{ll}
\mathrm{H}_{\mathrm{x}}^{\mathrm{E}_{\mathrm{x}}-\text { pol }} & \mathrm{H}_{\mathrm{y}}^{\mathrm{E}_{\mathrm{x}}-\mathrm{pol}} \\
\mathrm{H}_{\mathrm{x}}^{\mathrm{E}_{\mathrm{y}}-\text { pol }} & \mathrm{H}_{\mathrm{y}}^{\mathrm{E}_{\mathrm{y}}-\mathrm{pol}}
\end{array}\right)^{-1}\left(\begin{array}{c}
\mathrm{H}_{\mathrm{z}}^{\mathrm{E}_{\mathrm{x}}-\text { pol }} \\
\mathrm{H}_{\mathrm{z}}^{\mathrm{E}_{\mathrm{y}}-\text { pol }}
\end{array}\right)
$$

162 Meanwhile, HMTF $\mathbf{T}_{h} \in \mathbb{C}^{2 \times 2}$ is calculated from the horizontal magnetic field

163 components at an observation station and those at a reference station as follows:

$$
\mathbf{T}_{h}=\left(\begin{array}{cc}
\mathrm{T}_{\mathrm{xx}} & \mathrm{T}_{\mathrm{xy}} \\
\mathrm{T}_{\mathrm{yx}} & \mathrm{T}_{y y}
\end{array}\right)=\left(\begin{array}{ll}
\mathrm{H}_{\mathrm{x}}^{\mathrm{E}_{\mathrm{x}}-\text { pol }} & \mathrm{H}_{\mathrm{x}}^{\mathrm{E}_{\mathrm{y}}-\text { pol }} \\
\mathrm{H}_{\mathrm{y}}^{\mathrm{E}_{\mathrm{x}}-\text { pol }} & \mathrm{H}_{\mathrm{y}}^{\mathrm{E}_{\mathrm{y}}-\text { pol }}
\end{array}\right)\left(\begin{array}{ll}
\mathrm{H}_{\mathrm{r}, \mathrm{x}}^{\mathrm{E}_{\mathrm{x}}-\text { pol }} & \mathrm{H}_{\mathrm{r}, \mathrm{x}}^{\mathrm{E}_{\mathrm{y}}-\text { pol }} \\
\mathrm{H}_{\mathrm{r}, \mathrm{y}}^{\mathrm{E}_{\mathrm{x}}-\text { pol }} & \mathrm{H}_{\mathrm{r}, \mathrm{y}}^{\mathrm{E}_{\mathrm{y}}-\text { pol }}
\end{array}\right)^{-1},
$$

164 where the characters with subscript $r$ denote the magnetic field components at the

165 reference station. Further, PT $\boldsymbol{\Phi} \in \mathbb{R}^{2 \times 2}$ is calculated from the real and imaginary parts

166 of the impedance tensor as follows:

$$
\boldsymbol{\Phi}=\left(\begin{array}{ll}
\Phi_{11} & \Phi_{12} \\
\Phi_{21} & \Phi_{22}
\end{array}\right)=\left(\begin{array}{ll}
\operatorname{Re}\left(\mathrm{Z}_{\mathrm{xx}}\right) & \operatorname{Re}\left(\mathrm{Z}_{\mathrm{xy}}\right) \\
\operatorname{Re}\left(\mathrm{Z}_{\mathrm{yx}}\right) & \operatorname{Re}\left(\mathrm{Z}_{\mathrm{yy}}\right)
\end{array}\right)^{-1}\left(\begin{array}{ll}
\operatorname{Im}\left(\mathrm{Z}_{\mathrm{xx}}\right) & \operatorname{Im}\left(\mathrm{Z}_{\mathrm{xy}}\right) \\
\operatorname{Im}\left(\mathrm{Z}_{\mathrm{yx}}\right) & \operatorname{Im}\left(\mathrm{Z}_{\mathrm{yy}}\right)
\end{array}\right)
$$

167 where $Z_{\mathrm{xx}}, \mathrm{Z}_{\mathrm{xy}}, \mathrm{Z}_{\mathrm{yx}}$, and $\mathrm{Z}_{\mathrm{yy}}$ are the components of the impedance tensor $\mathbf{Z} \in \mathbb{C}^{2 \times 2}$, which 168 is computed as follows:

$$
\left(\begin{array}{ll}
Z_{x x} & Z_{x y} \\
Z_{y x} & Z_{y y}
\end{array}\right)=\left(\begin{array}{ll}
E_{x}^{E_{x}-\text { pol }} & E_{x}^{E_{y}-\text { pol }} \\
E_{y}^{E_{x}-\text { pol }} & E_{y}^{E_{y}-\text { pol }}
\end{array}\right)\left(\begin{array}{ll}
H_{x}^{E_{x}-\text { pol }} & H_{x}^{E_{y}-\text { pol }} \\
H_{y}^{E_{x}-\text { pol }} & H_{y}^{E_{y}-p o l}
\end{array}\right)^{-1} .
$$

169 NMTRF $\mathbf{Y}=\left(\mathrm{Y}_{\mathrm{x}}, \mathrm{Y}_{\mathrm{y}}\right)^{T} \in \mathbb{C}^{2}$ is defined as the frequency response function between the 170 voltage difference $\mathrm{V} \in \mathbb{C}$ for the dipole of an NMT station and the horizontal magnetic

171 field components at a reference station (Uyeshima et al. 2001). 


$$
\mathrm{V}=\mathrm{Y}_{\mathrm{x}} \mathrm{H}_{\mathrm{x}}+\mathrm{Y}_{y} \mathrm{H}_{\mathrm{y}}
$$

172 In the developed combined inversion scheme, voltage differences were computed using

173 an algorithm similar to that proposed by Siripunvaraporn et al. (2004). Because long

174 dipoles of the NMT method generally extend over multiple finite elements of the

175 computational mesh, dipoles are divided into segments by element edges (Figure 1).

176 Each segment is located within an element surface, which is triangular. The voltage

177 difference along a dipole can be obtained by summing the voltage differences of the

178 small segments. According to Faraday's law with the $\mathrm{e}^{-\mathrm{i} \omega t}$ time dependence, the voltage

179 difference $d V \in \mathbb{C}$ along each segment (illustrated as a red dashed line in Figure 1) can

180 be calculated as follows:

$$
d V=-E_{1} L_{1}-E_{2} L_{2}-i \omega \mu_{0} H_{n} S
$$

181 where $E_{1}$ and $E_{2}$ are the electric field components along the triangle edges, $L_{1}$ and $L_{2}$

182 are the lengths of the edges, $\mathrm{i}$ is the imaginary unit, $\mathrm{H}_{\mathrm{n}}$ is the magnetic field

183 component normal to the triangle, and $\mathrm{S}$ is the area of the triangle. The magnetic field

184 component normal to triangle $\mathrm{H}_{n}$ is calculated from the electric field components of the

185 element edges on the Earth's surface using Faraday's law (Equation (6)). Based on the 
187 can be calculated as follows:

$$
\mathbf{Y}=\left(\begin{array}{c}
Y_{x} \\
Y_{y}
\end{array}\right)=\left(\begin{array}{ll}
H_{x}^{E_{x}-\text { pol }} & H_{y}^{E_{x}-\text { pol }} \\
H_{x}^{E_{y}-\text { pol }} & H_{y}^{E_{y}-\text { pol }}
\end{array}\right)^{-1}\left(\begin{array}{c}
V^{E_{x}-p o l} \\
V^{E_{y}-\text { pol }}
\end{array}\right)=\mathbf{H}^{-1} \mathbf{V}
$$

188 In the combined inversion, we find the model parameters (subsurface electrical

189 resistivities) that minimize the objective function $\phi(\mathbf{m}) \in \mathbb{R}:$

$$
\phi(\mathbf{m})=\|\mathbf{W} \mathbf{d}-\mathbf{W F}(\mathbf{m})\|^{2}+\alpha^{2}\|\mathbf{R m}\|^{2},
$$

190 where $\mathbf{W}$ is the diagonal matrix of the diagonals that are reciprocals of the errors in the

191 observed data; $\mathbf{d}$ and $\mathbf{F}(\mathbf{m})$ are the real vectors containing the observed and calculated

192 response functions, respectively; and $\mathbf{R}$ denotes the roughening matrix. Vectors $\mathbf{d}$ and

$193 \mathbf{F}(\mathbf{m})$ contain the real and imaginary parts of the complex frequency response functions

194 separately. The roughening matrix provides the differences in the log-resistivities of the

195 adjacent parameter cells $\mathbf{r}$ as follows:

$$
\begin{gathered}
\mathbf{R m}=\mathbf{r}=\left(\begin{array}{lllll}
r_{1} & \cdots & r_{i} & \cdots & r_{M}
\end{array}\right)^{T} \\
r_{i}=F_{i} m_{i}-\sum_{j=1}^{F_{i}} m_{j, i}
\end{gathered}
$$

196 where $\mathrm{M}$ is the number of model parameters; $\mathrm{F}_{\mathrm{i}}$ and $\mathrm{m}_{\mathrm{i}}$ are the number of faces and

197 the log-resistivity of the $\mathrm{i}$-th parameter cell, respectively; and $\mathrm{m}_{\mathrm{j}, \mathrm{i}}$ is the log-resistivity 
198 of the parameter cell adjacent to the $\mathrm{i}$-th parameter cell through its $\mathrm{j}$-th face. It should

199 be noted that the objective function (Equation (14)) does not include the prior model,

200 unlike the previous inversion schemes that use the response functions free from

201 galvanic distortion (Patro et al. 2013; Tietze et al. 2015; Campanyà et al. 2016).

202 Therefore, our inversion scheme can estimate subsurface electrical resistivity without

203 dependency on the prior model. After linearizing Equation (14), the model parameters

204 are calculated by the data-space Gauss-Newton method, as described in Usui et al.

205 (2017).

206

207 The Gauss-Newton method requires a sensitivity matrix (Jacobian matrix), which

208 comprises the partial derivatives of the response functions with respect to the model

209 parameters. The sensitivity matrix is computed using the reciprocity property of the

210 electromagnetic field (Rodi 1976). Because the voltage difference and the horizontal

211 magnetic field used for calculating NMTRF can be expressed as linear combinations of

212 the electric field along the element edges, which are the unknowns in Equation (5), the

213 sensitivity matrix for NMTRF can be calculated using the reciprocity approach (Rodi 
214 1976). While Siripunvaraporn et al. (2004) used only the derivatives of the voltage

215 differences for calculating the sensitivity matrix, we consider the derivatives of the

216 reference horizontal magnetic field components in addition to the derivatives of the

217 voltage differences. Thus, the derivatives of NMTRF can be expressed as follows:

$$
\frac{\partial \mathbf{Y}}{\partial \mathbf{m}}=\frac{\partial \mathbf{H}^{-1}}{\partial \mathbf{m}} \mathbf{V}+\mathbf{H}^{-1} \frac{\partial \mathbf{V}}{\partial \mathbf{m}}
$$

\section{3. Synthetic inversion analysis and discussion}

220 We performed inversions using two different datasets. The first dataset contains PT,

221 VMTF, and HMTF, whereas the second dataset contains PT, VMTF, HMTF, and

222 NMTRF. Hereon, we refer to the former and latter cases as Comb-A and Comb-B,

223 respectively. We performed a synthetic inversion analysis to assess whether the two new

224 types of the combined inversion approaches can reproduce the appropriate resistivity

225 structure robustly. The target model used in this study was the oblique conductor (OC)

226 model used by Tietze et al. (2015). However, we exchanged the $\mathrm{x}$ - and $\mathrm{y}$-axes to have

227 the strike of the regional 2-D structure of the model parallel to the X-axis. PT and

228 VMTF were calculated for the 100 observation stations with the exact locations used by 
229 Tietze et al. (2015). The HMTF was also calculated for the same observation stations,

230 except for the bottom-right point in Figures 2 and 3 (the station at $\mathrm{x}=-18 \mathrm{~km}$ and $\mathrm{y}=18$

$231 \mathrm{~km})$. We used the bottom-right point as the reference station to calculate the HMTF. In

232 addition, we calculated NMTRF at the 36 NMT observation stations (Figure 3 ). The

233 NMT observation stations are represented as broken lines with circular ends, as shown

234 in Figure 3. The dipole length of each NMT station is $5.66 \mathrm{~km}$. We used the horizontal

235 magnetic field at the bottom-right point as the reference magnetic field to calculate

236 NMTRF in the same manner as the HMTF. We calculated PT, VMTF, and HMTF for 16

237 logarithmically spaced periods from 0.01 to $1,000 \mathrm{~s}$, as in Tietze et al. (2015).

238 Meanwhile, we calculated NMTRF for ten logarithmically spaced periods from 10 to

$23910,000 \mathrm{~s}$ because it is usually difficult to obtain the response functions at short periods

240 at NMT stations. Because NMTRF can be estimated with small errors at periods longer

241 than several thousand seconds, as indicated in the Introduction section, we set the

242 maximum period for NMTRF to be longer than the other response functions. Because it

243 is difficult to incorporate a thin $0.1 \mathrm{~km}$ near-surface layer $(100 \Omega \mathrm{m})$ of the OC model

244 using a tetrahedral mesh, which requires a huge number of elements to represent such a 
245 thin layer, we used a forward program using hexahedral mesh (Usui 2020; Usui et al.

$2462020)$ to calculate the synthetic response functions. The algorithm for the forward

247 calculation using the hexahedral mesh is the same as that described in Section 2. By

248 using different meshes, we can also avoid the so-called "inverse crime." Before

249 calculating PT from the impedance tensor, we provided a galvanic distortion to the

250 calculated impedance tensor by multiplying a distortion tensor because the impedance

251 tensor observed in the real world is generally distorted. The distortion tensors were

252 calculated by randomly generated values of the twist angle (within $\pm 30^{\circ}$ ), shear angle

253 (within $\pm 30^{\circ}$ ), anisotropy (within \pm 0.5 ), and the gains (from 0.1 to 10.0 in the

254 logarithmic axis) of the Groom-Bailey decomposition (Groom and Bailey 1989).

255

256 To generate synthetic data, we added Gaussian noise with a mean of zero to the

257 response functions. The standard deviation of the noise for VMTF was 0.02 , as used in

258 Tietze et al. (2015). Similarly, the standard deviation of the noise for HMTF was 0.02.

259 The standard deviations for NMTRF were $3 \%$ of their absolute values; that is, $0.03\left|\mathrm{Y}_{\mathrm{x}}\right|$

260 or $0.03\left|\mathrm{Y}_{\mathrm{y}}\right|$. For PT, we added Gaussian noise to the distorted impedance tensor and 
then calculated the PT errors by error propagation, as indicated in Patro et al. (2013).

262 The standard deviation of the noise to the impedance tensor was $3 \%$ for each

263 component, along with a floor of $3 \%$ of $\left|\mathrm{Z}_{\mathrm{xy}} \mathrm{Z}_{\mathrm{yx}}\right|^{1 / 2}$ for diagonals, following Tietze et

264 al. (2015). We started the combined inversion from three different uniform half-space

265 models $(10,100$, and $1,000 \Omega \mathrm{m})$ to investigate the dependency of the inversion results

266 on the initial model. In all cases, the tradeoff parameter $\alpha$ was set to 10 .

267

268 The resultant resistivity structures of Comb-A are shown in Figure 2. The estimated

269 resistivity structures have characteristic resistivity anomalies in the OC model.

270 Specifically, both the resistivity contrast along the y-direction and the oblique conductor

271 could be adequately imaged. However, the estimated resistivity values tended to be

272 larger than the actual values of the OC model. It should be noted that, despite using

273 significantly different initial models, no significant differences were observed in the

274 resultant resistivity structure down to $15 \mathrm{~km}$ below ground. Tietze et al. (2015)

275 performed a combined inversion of PT and VMTF with different prior and initial

276 models between $10 \Omega \mathrm{m}$ and $1000 \Omega \mathrm{m}$ (the prior model was identical to the initial model 
277 in each case). Although the target model (i.e., OC model) was the same as that used in

278 this study, Tietze et al. (2015) indicated a more significant dependency of the resultant

279 resistivity structure on the prior and initial models, which is inconsistent with our

280 results. Even if we performed the combined inversion of PT and VMTF, just like Tietze

281 et al. (2015), the resultant resistivity structure down to $15 \mathrm{~km}$ below ground presented

282 no significant dependency on the initial models as the Comb-A results (Figure S1 in

283 Supporting Information). Therefore, this inconsistency was not attributed to the use of

284 HMTF. Because the inversion scheme of this study does not use the prior model, unlike

285 Tietze et al. (2015), the robustness of the results of our combined inversions is caused

286 by not using the prior model. Other studies using PT and/or the magnetic transfer

287 functions demonstrated a deficiency of robustness owing to the low sensitivity to the

288 absolute values of the resistivity structure (Patro et al. 2013; Tietze et al. 2015;

289 Campanyà et al. 2016). However, the results of our synthetic inversions suggest that not

290 using the prior model can increase the robustness of the inversion using PT and the

291 magnetic transfer functions. 
293 Figure 3 presents the resultant resistivity structures of Comb-B down to $15 \mathrm{~km}$ below

294 ground. Similar to Comb-A (Figure 2), only a slight difference is observed among the

295 resultant resistivity structures starting from the different initial models. Characteristic

296 resistivity anomalies of the OC model can also be adequately recovered by Comb-B.

297 Upon comparing the results of Comb-A (Figure 2) and Comb-B (Figure 3), Comb-B

298 recovered the resistivity values at depths of $5 \mathrm{~km}$ and $15 \mathrm{~km}$ closer to the actual values

299 of the OC model. It is considered that the additional use of NMTRF increased the

300 sensitivity to the absolute values of the resistivity structure, allowing us to obtain a

301 more reliable resistivity structure.

302

303 Figure 4 illustrates the electrical resistivity variation down to a depth of $100 \mathrm{~km}$ at the

304 two points of $(x=10 \mathrm{~km}, \mathrm{y}=-10 \mathrm{~km})$ and $(x=-10 \mathrm{~km}, \mathrm{y}=10 \mathrm{~km})$. The locations of the two

305 points are symmetrical about the axis of the oblique conductor. The former point is

306 located at the resistive side, whereas the latter is located at the conductive side.

307 Although both Comb-A and Comb-B were unable to recover sharp resistivity changes at

308 a depth of $70 \mathrm{~km}$, this was inevitable owing to the smoothing effect due to the 
309 stabilizing term of the objective function (Rodi and Macki 2012). At depths greater than

$31015 \mathrm{~km}$, apparent differences are observed between the resultant resistivity structures of

311 Comb-A and Comb-B. Figure 4 demonstrates that the resistivity values obtained by

312 Comb-A tend to be larger than the actual values of the OC model at the depths down to

$313100 \mathrm{~km}$. Furthermore, the overestimation of the resistivity becomes more significant

314 with an increase in the initial resistivity value. This overestimation demonstrates that the

315 results of the combined inversion using PT and the magnetic transfer function depend

316 on the initial model, especially in the deep parts, where the sensitivity of the data is

317 relatively low. Compared to the Comb-A results, the resistivity values obtained by

318 Comb-B are closer to the actual values. Although the dependency on the initial model

319 can also be found in the Comb-B results, no significant bias exists from the actual

320 model, unlike the results of Comb-A. Especially at depths greater than $70 \mathrm{~km}$, the

321 resistivity values of Comb-B converge to the actual value (100 $\Omega \mathrm{m})$ without depending

322 on the initial model. It is believed that the use of NMTRF increased the robustness

323 against the initial models and enabled us to estimate a more accurate resistivity structure

324 because NMTRF has a significant sensitivity to the absolute values of the subsurface 
325 resistivity as the impedance tensor.

326

327 In addition, we found notable differences in the stabilization of the inversion between

328 Comb-A and Comb-B (Figure 5). Figure 5 compares the variations in the objective

329 functions during the combined inversions. At the 0 -th iteration, the objective functions

330 of Comb-B varied according to the initial model, while those of Comb-A presented

331 similar values without a dependency on the initial model. Because the objective

332 function at the 0 -th iteration was calculated from the initial model, the variation of the

333 objective function at the 0 -th iteration reflects the high sensitivity of NMTRF to the

334 absolute values of the subsurface resistivity structure. In the Comb-B inversions, the

335 objective functions decreased rapidly and remained unchanged up to the 7-th iteration.

336 Meanwhile, in Comb-A, the convergence of the inversion significantly depended on the

337 initial models. While the objective functions remained unchanged after the 5 -th iteration

338 when the initial resistivity was $10 \Omega \mathrm{m}$, it required up to the 16 -th iteration to converge

339 when we used the initial model of $1,000 \Omega \mathrm{m}$. When starting from the same initial

340 model, Comb-A required more iterations than Case B. The relatively low sensitivity to 
341 the absolute values of the subsurface resistivity apparently led to the instability in

342 Comb-A. Figure 5 demonstrates that the addition of NMTRF can improve the inversion

343 stability.

344

345 Because both Comb-A and Comb-B were able to recover the characteristic features of

346 the target resistivity structure, we can conclude that both combined inversion

347 approaches are effective in estimating the subsurface resistivity structure. This study

348 indicates that combined inversions using distortion-free response functions have the

349 potential to estimate subsurface resistivity more robustly than what was previously

350 thought. However, an overestimation bias in the resultant resistivity structure and poor

351 convergence of the Gauss-Newton iteration was found in the results of Comb-A. The

352 low sensitivity to the absolute values of the subsurface resistivity is thought to be the

353 cause of these problems. Because both issues were improved by Comb-B, we confirmed

354 that the combined inversion using NMTRF can enhance the reliability of the resultant

355 resistivity structure and the stability of the inversion. Therefore, we believe that the

356 combined use of NMTRF is the preferred approach for robustly estimating the 
357 resistivity structure.

358

\section{4. Summary}

360 To evaluate the effectiveness of the two new types of 3-D combined inversion using

361 response functions free from galvanic distortion, we performed synthetic inversion

362 analyses using a newly developed 3-D combined inversion scheme; this can include

363 NMTRF as the datasets in addition to the response functions of the usual NMT

364 measurements. By comparing the estimated resistivity structures and histories of the

365 objective functions, we obtained the following results:

366 1. Despite using only PT, VMTF, and HMTF, the combined inversion recovered the

367 characteristic resistivity anomalies of the target model in the shallow part without a

368 significant dependence on the initial models. This suggests that the combined

369 inversion using only PT and the magnetic transfer functions has the potential to

370 recover the subsurface resistivity structure more robustly than what was previously

371 thought if the prior model is not used.

372 2. When we used NMTRF in addition to PT and the magnetic transfer functions, the 
373 resultant resistivity structure became closer to the actual model. Therefore, we

374 confirmed that the additional use of NMTRF enabled us to estimate a more accurate

$375 \quad$ resistivity structure.

376 3. The objective functions stably decreased when NMTRF is included as data. In

377 contrast, the convergence of the inversion was worse and depending significantly on

378 the initial model when we used only PT, VMTF, and HMTF. This difference

379 demonstrates that the use of NMTRF can improve the stability of the combined

380 inversion.

381 Because PT, VMTF, and HMTF can be calculated from the observed data of the MT

382 measurements, we can apply the combined inversion proposed in this study to existing

383 data. In addition, in many areas of Japan, NMT measurements have been conducted in

384 the same survey area as usual MT measurements. This enables us to perform the

385 inversion by combining NMTRF with other distortion-free response functions. The

386 aforementioned results suggest that the combined inversion using only the response

387 function free of galvanic distortion has the potential to robustly recover the target

388 resistivity structure, especially by using NMTRF. Therefore, we expect that the 
389 developed combined inversion approach will help in robustly recovering resistivity

390 structures by avoiding the distortions caused by near-surface anomalies.

392 Declarations

\section{Consent for publication}

Not applicable measurement; RMS, root mean square

\section{Availability of data and materials}


The authors declare that they have no competing interests.

\section{Funding}

408

Not applicable

\section{Authors' contributions}

YU developed a combined inversion scheme and performed synthetic

inversion tests. MU is the inventor of the combined inversion of the phase tensor, magnetic transfer functions, and Network-MT response function. All authors read and approved the final manuscript.

\section{Acknowledgments}

In this study, we used the computer systems of the Earthquake and

Volcano Information Center of the Earthquake Research Institute, the tools (Wessel et al. 2013). 


\section{References}

426 Caldwell TG, Bibby HM, Brown C (2004) The magnetotelluric phase tensor. Geophys J

427 Int 158:457-469. doi:10.1111/j.1365-246X.2004.02281.X

428 Campanyà $\mathrm{J}$, Ogaya $\mathrm{X}$, Jones AG et al. (2016) The advantages of complementing MT

429 profiles in 3-D environments with geomagnetic transfer function and interstation

430 horizontal magnetic transfer function data: Results from a synthetic case study. Geophys

431 J Int 207:1818-1836. doi:10.1093/gji/ggw357

432 Egbert GD, Kelbert A (2012) Computational recipes for electromagnetic inverse

433 problems. Geophys J Int 189:251-267. doi:10.1111/j.1365-246X.2011.05347.x

434 Groom RW, Bailey RC (1989) Decomposition of magnetotelluric impedance tensors in

435 the presence of local three-dimensional galvanic distortion. J Geophys Res 94:1913- 
437 Jin J (2002) The finite element method in electromagnetics, 2nd edn. John Wiley \&

438 Sons, New York

439 Kelbert A, Meqbel N, Egbert GD, Tandon K (2014) ModEM: A modular system for

440 inversion of electromagnetic geophysical data. Comput Geosci 66:40-53.

441 doi:10.1016/j.cageo.2014.01.010

442 Kinoshita M, Uyeshima M, Uyeda S (1989) Earthquake prediction research by means of

443 telluric potential monitoring, progress report no. 1: Installation of monitoring network.

444 Bulletin of the Earthquake Research Institute, 64:255-231

445 Ogawa Y (2002) On two-dimensional modeling of magnetotelluric field data. Surv

446 Geophys 23:251-273. doi:10.1023/A:1015021006018

447 Patro PK, Uyeshima M, Siripunvaraporn W (2013) Three-dimensional inversion of

448 magnetotelluric phase tensor data. Geophys J Int 192:58-66. doi:10.1093/gji/ggs014

449 Rodi WL (1976) A technique for improving the accuracy of finite element solutions for

450 magnetotelluric data. Geophys J Int 44:483-506. doi:10.1111/j.1365- 
452 Rodi WL, Mackie R (2012) The inversion problem. In: Chave AD, Jones AG (eds) The

453 magnetotelluric method: Theory and practice Cambridge University Press, Cambridge

454 Simpson F, Bahr K (2005) Practical magnetotellurics Cambridge University Press,

455 Cambridge

456 Siripunvaraporn W, Egbert G (2009) WSINV3DMT: Vertical magnetic field transfer

457 function inversion and parallel implementation. Phys Earth Planet Inter 173:317-329.

458 doi: $\underline{10.1016 / j . p e p i .2009 .01 .013}$

459 Siripunvaraporn W, Egbert G, Lenbury Y, Uyeshima M (2005) Three-dimensional

460 magnetotelluric inversion: Data-space method. Phys Earth Planet Inter 150:3-14.

461 doi: $\underline{10.1016 / j . p e p i .2004 .08 .023}$

462 Siripunvaraporn W, Uyeshima M, Egbert G (2004) Three-dimensional inversion for

463 Network-magnetotelluric data. Earth Planets Space 56:893-902.

464 doi:10.1186/BF03352536

465 Tietze K, Ritter O, Egbert GD (2015) 3-D joint inversion of the magnetotelluric phase

466 tensor and vertical magnetic transfer functions. Geophys J Int 203:1128-1148. 
468 Usui Y (2015) 3-D inversion of magnetotelluric data using unstructured tetrahedral

469 elements: Applicability to data affected by topography. Geophys J Int 202:828-849.

470 doi:10.1093/gii/ggv186

471 Usui Y (2020) Three-dimensional combined inversion scheme of the wideband-

472 magnetotelluric method and the Network-MT method, SGEPSS Fall Meeting 2020.

473 Online, 1-4 November 2020

474 Usui Y, Ogawa Y, Aizawa K et al. (2017) Three-dimensional resistivity structure of

475 Asama Volcano revealed by data-space magnetotelluric inversion using unstructured

476 tetrahedral elements. Geophys J Int 208:1359-1372. doi: 10.1093/gji/ggw459

477 Usui Y, Uyeshima M, Ogawa T et al. (2020) Resistivity structure around the Atotsugawa

478 fault system revealed by inversion schemes combining wideband- and Network-MT

479 methods, JpGU-AGU Joint Meeting. Online, 24-28 May 2020

480 Usui Y, Uyeshima M, Ogawa T et al. (2021) Electrical resistivity structure around the

481 Atotsugawa fault, central Japan, revealed by a new 2-D inversion method combining

482 wideband-MT and Network-MT data sets. J Geophys Res Solid Earth 126:e2020. 
484 Uyeshima M (2007) EM monitoring of crustal processes including the use of the

485 Network-MT observations. Surv Geophys 28:199-237. doi:10.1007/s10712-007-9023-X

486 Uyeshima M, Kinoshita M, Iino H, Uyeda S (1989) Earthquake prediction research by

487 means of telluric potential monitoring, progress report no. 2: Preliminary study on

488 Teshikaga channel 2 signals and the seismicity in the region off Kushiro. Bull Earthq

489 Res Inst Univ Tokyo, 64:487-515

490 Uyeshima M, Utada H, Nishida Y (2001) Network-magnetotelluric method and its first

491 results in central and eastern Hokkaido, NE Japan. Geophys J Int 146:1-19,

$492 \quad \underline{10.1046 / \mathrm{j} .0956-540 \mathrm{x} .2001 .01410 . \mathrm{x}}$

493 Wessel P, Smith WHF, Scharroo R et al. (2013) Generic mapping tools: Improved

494 version released, Trans Am Geophys Un. 94:409-410. doi: 10.1002/2013EO450001 


\section{Figure legends:}

497 Figure 1. Schematic view illustrating how to calculate the voltage difference along each

498 dipole of the NMT observation station. Here, $E_{1}$ and $E_{2}$ are the electric field

499 components along the edges of the triangle, $\mathrm{L}_{1}$ and $\mathrm{L}_{2}$ are the lengths of the edges, $\mathrm{i}$ is

500 the imaginary unit, $\mu_{0}$ is the magnetic permeability of vacuum, $H_{n}$ is the magnetic

501 field component normal to the triangle, and $\mathrm{S}$ is the area of the triangle. The solid lines

502 indicate the element edges, while a thick dash line denotes a dipole of the NMT

503 observation station. The black-filled circle denotes the endpoints of the dipole. The

504 voltage difference along each dipole is computed by summing the voltage differences

$505 \mathrm{dV}$ of the small segments.

506

507 Figure 2. Electrical resistivity structures obtained by Comb-A. (a) True resistivity

508 structure (OC model). (b)-(c) Resultant resistivity structures obtained by the combined

509 inversion with three different initial models. The lowermost panels are the vertical

510 cross-sections of the profile along the major axis of the oblique conductor, which is

511 shown as a white line in the upper-left panel. The inverted triangles indicate the 
512 locations of observation stations. We used the bottom-right point (the station at $\mathrm{x}=-18$

$513 \mathrm{~km}$ and $\mathrm{y}=18 \mathrm{~km}$ ) as the reference station for calculating HMTF.

514

515 Figure 3. Electrical resistivity structures obtained by Comb-B. (a) True resistivity

516 structure (OC model). (b)-(c) Resultant resistivity structures obtained by the combined

517 inversion with three different initial models. The lowermost panels indicate the vertical

518 cross-sections of the profile along the major axis of the oblique conductor, which is

519 shown as a white line in the upper-left panel. The inverted triangles indicate the

520 locations of observation stations of PT, VMTF, and HMTF. The broken lines with

521 circular ends indicate the dipoles of the NMT stations. We used the bottom-right point

522 (the station at $\mathrm{x}=-18 \mathrm{~km}$ and $\mathrm{y}=18 \mathrm{~km}$ ) as the reference station for calculating HMTF

523 and NMTRF.

524

525 Figure 4. Electrical resistivity variation with a depth at (a) $(\mathrm{x}, \mathrm{y})=(10 \mathrm{~km},-10 \mathrm{~km})$ and

526 (b) $(x, y)=(-10 \mathrm{~km}, 10 \mathrm{~km})$. The thick black lines indicate the resistivity variation of

527 the true resistivity structure. The broken colored and solid-colored lines indicate the 
528 resistivity variations of the resistivity structure obtained by Comb-A and Comb-B,

529 respectively.

530

531 Figure 5. Objective function versus the iteration number for combined inversions with

532 different initial models. The broken and solid lines indicate the objective function

533 changes during Comb-A and Comb-B inversions. 


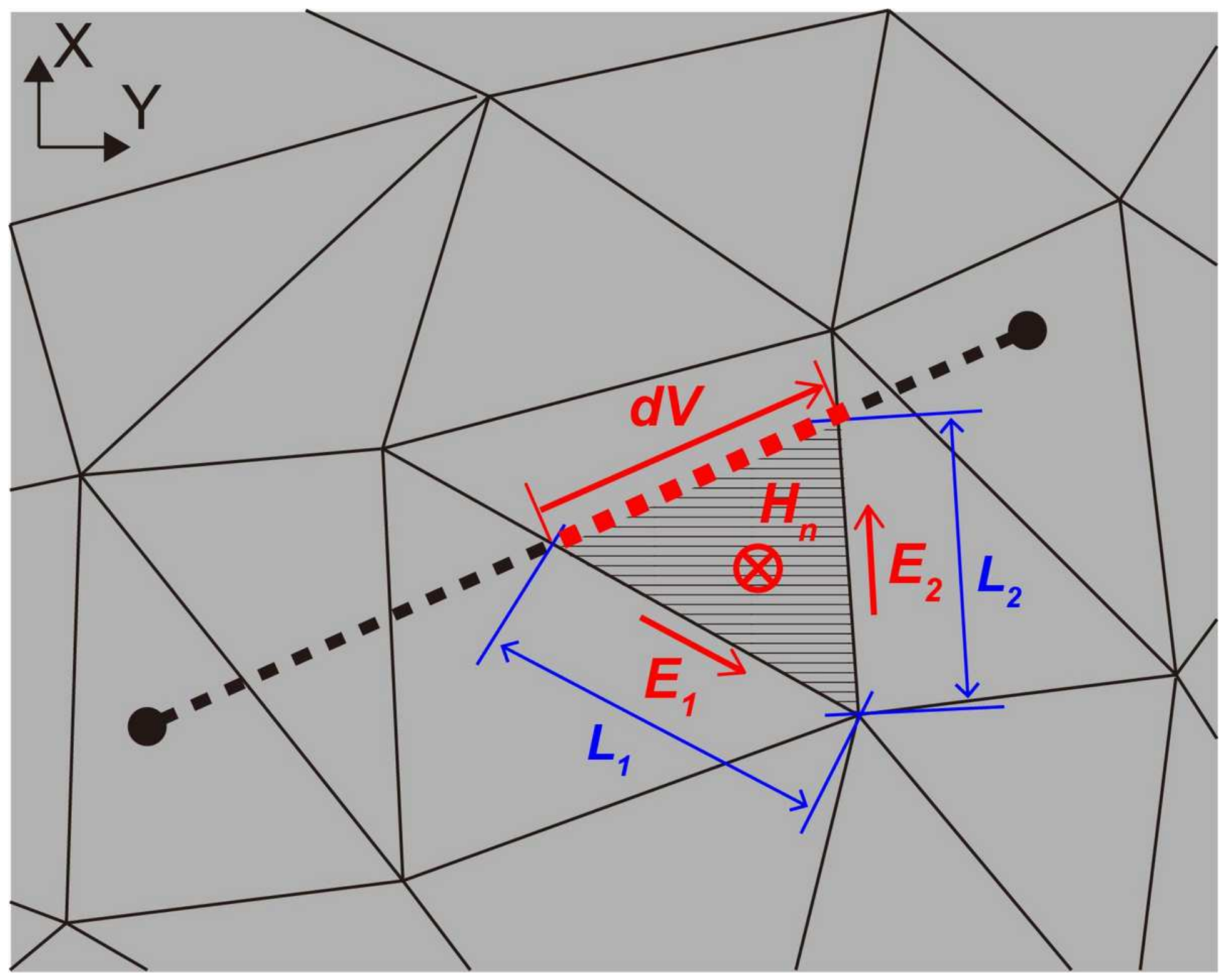

\section{Figure 1}

Schematic view illustrating how to calculate the voltage difference along each dipole of the NMT observation station. Here, E1 and E2 are the electric field components along the edges of the triangle, L1 and $L 2$ are the lengths of the edges, $i$ is the imaginary unit, $\mu 0$ is the magnetic permeability of vacuum, $\mathrm{Hn}$ is the magnetic field component normal to the triangle, and $\mathrm{S}$ is the area of the triangle. The solid lines indicate the element edges, while a thick dash line denotes a dipole of the NMT observation station. The black-filled circle denotes the endpoints of the dipole. The voltage difference along each dipole is computed by summing the voltage differences $\mathrm{dV}$ of the small segments. 
(a) True resistivity structure
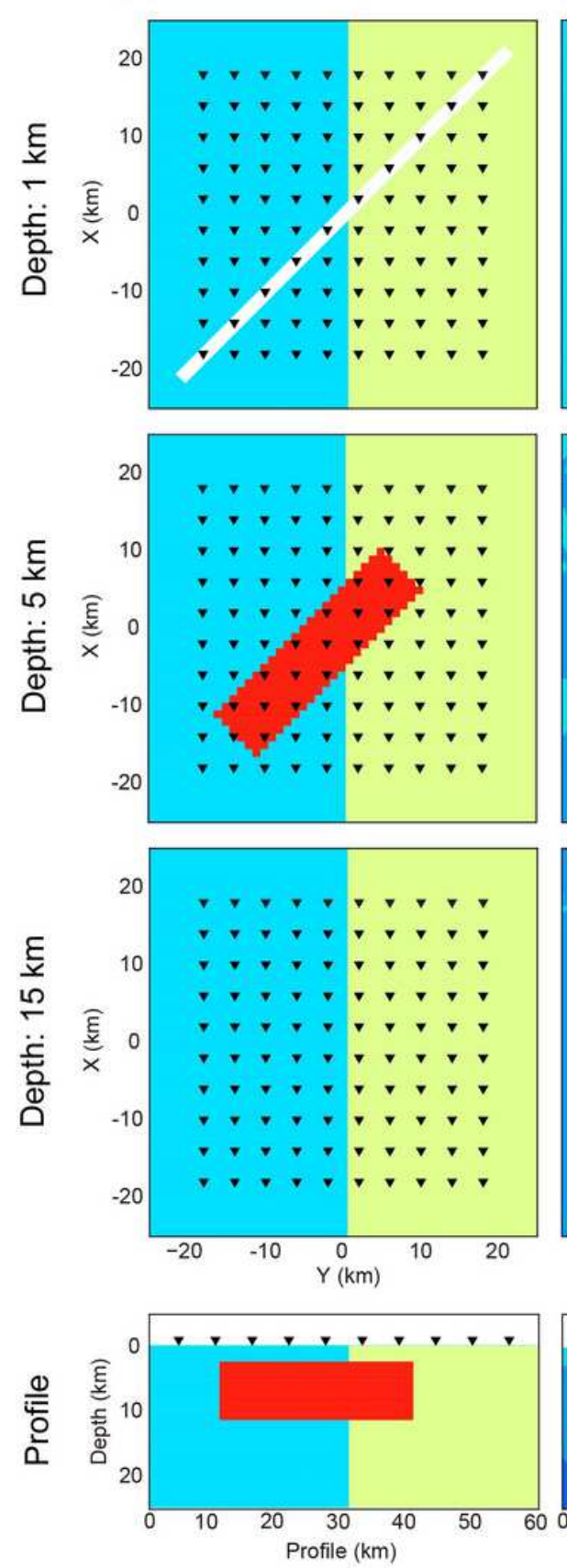

(b) From $10 \Omega \mathrm{m}$
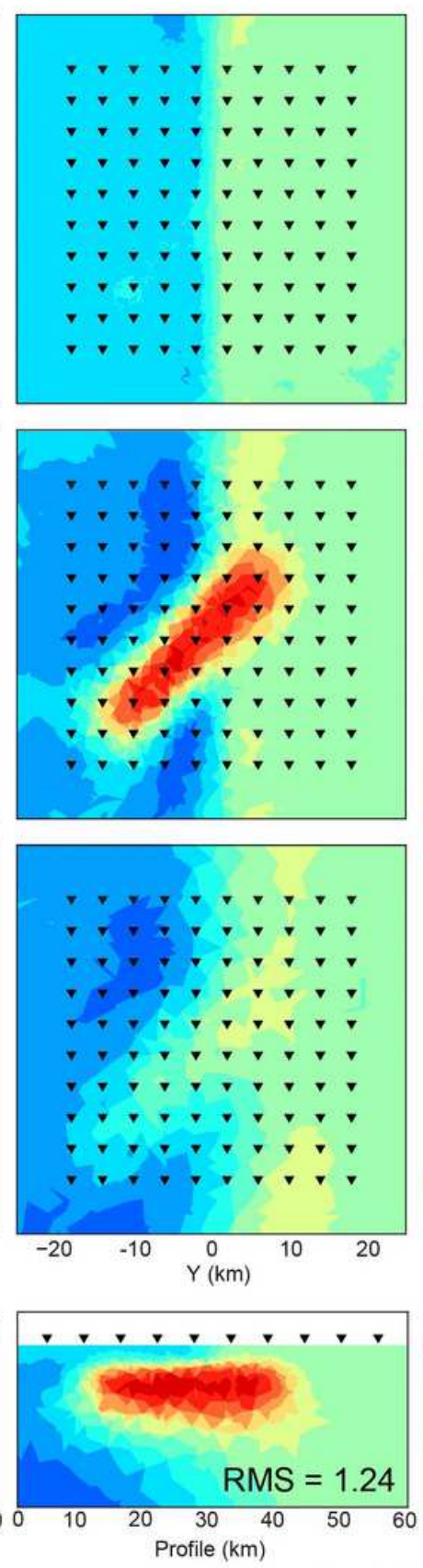

(c) From $100 \Omega \mathrm{m}$
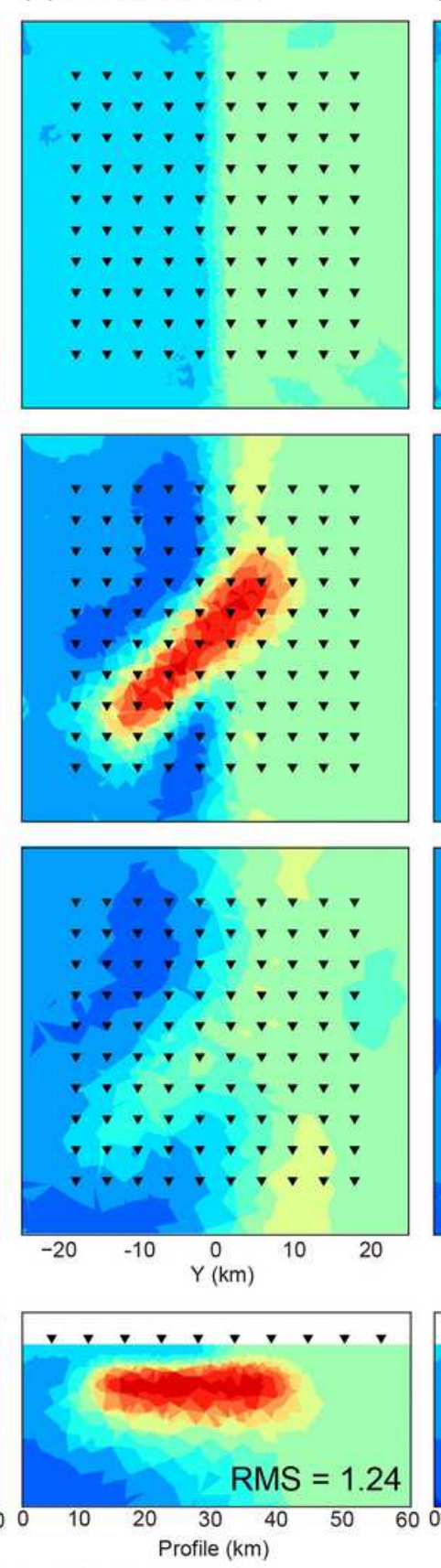

(d) From $1000 \Omega \mathrm{m}$
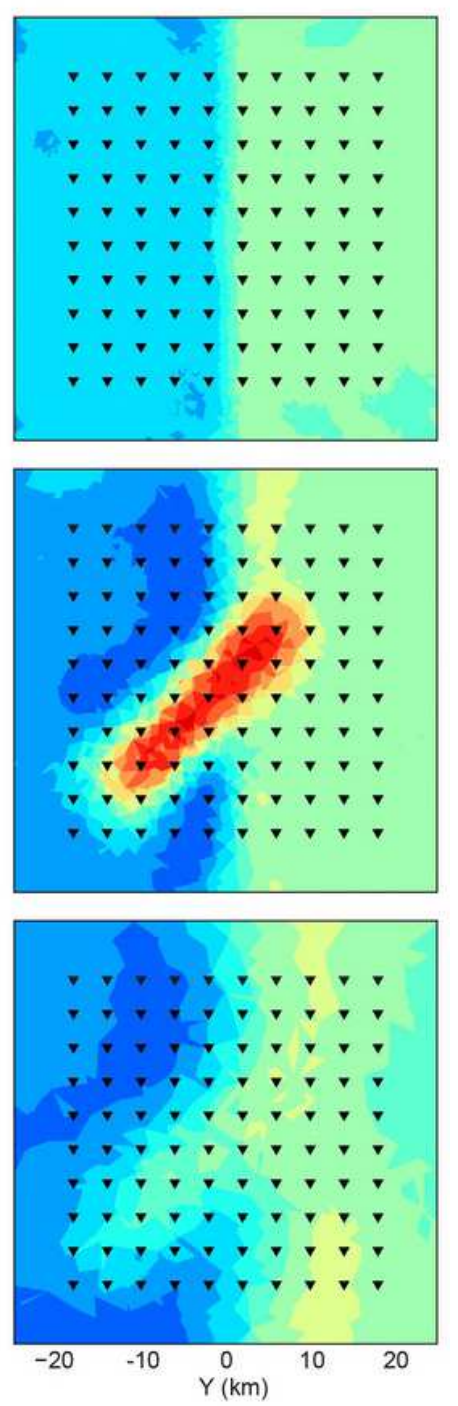

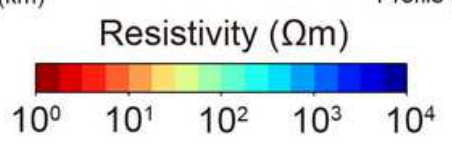

\section{Figure 2}

Electrical resistivity structures obtained by Comb-A. (a) True resistivity structure (OC model). (b)-(c) Resultant resistivity structures obtained by the combined inversion with three different initial models. The lowermost panels are the vertical cross-sections of the profile along the major axis of the oblique conductor, which is shown as a white line in the upper-left panel. The inverted triangles indicate the locations of observation stations. We used the bottom-right point (the station at $x=-18 \mathrm{~km}$ and $\mathrm{y}=18 \mathrm{~km}$ ) as the reference station for calculating HMTF. 
(a) True resistivity structure

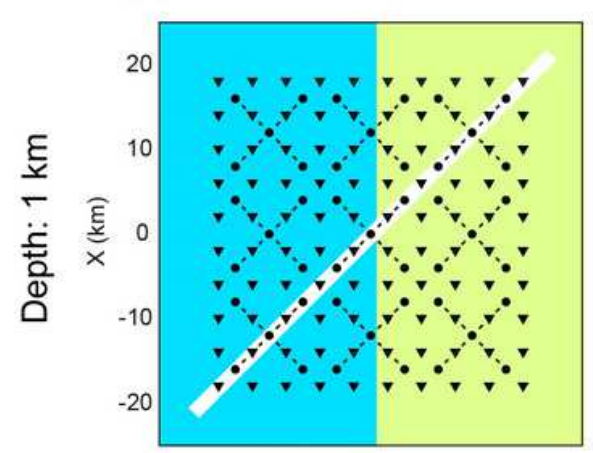

(b) From $10 \Omega \mathrm{m}$
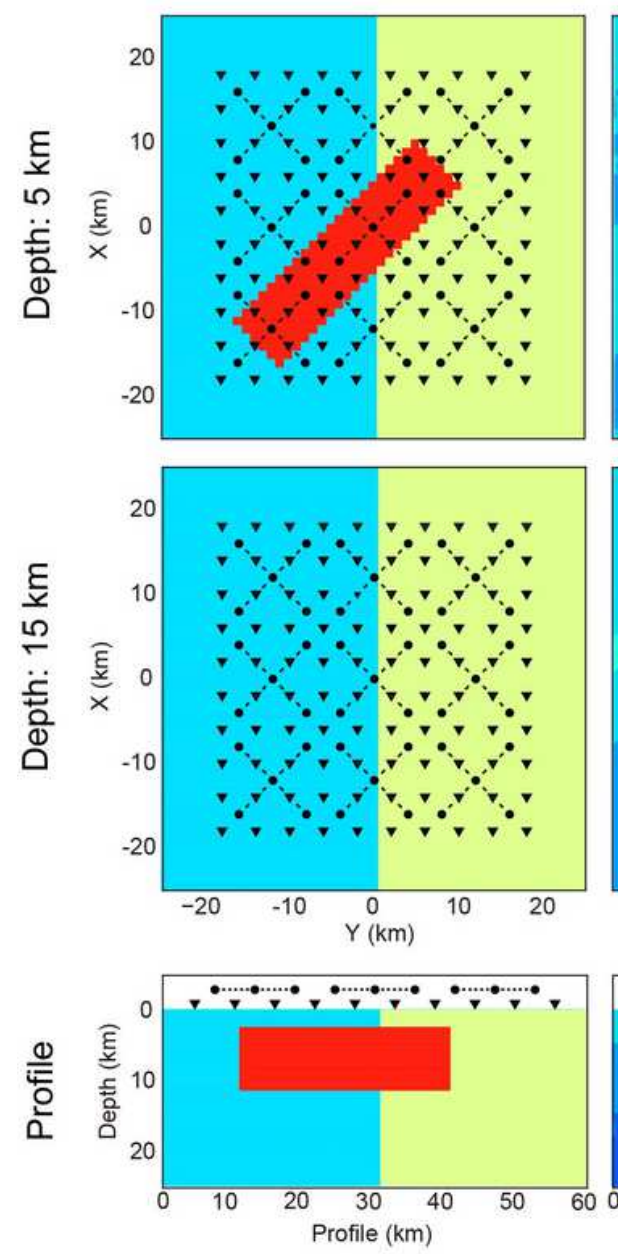

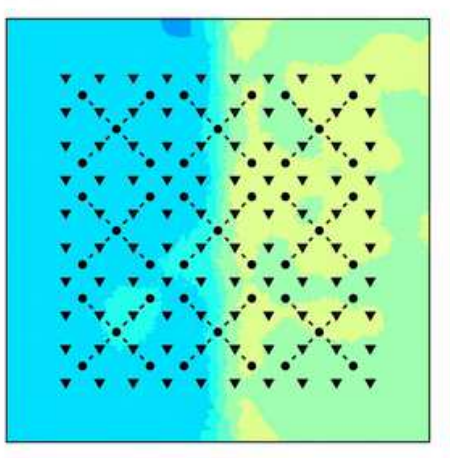

(c) From $100 \Omega \mathrm{m}$
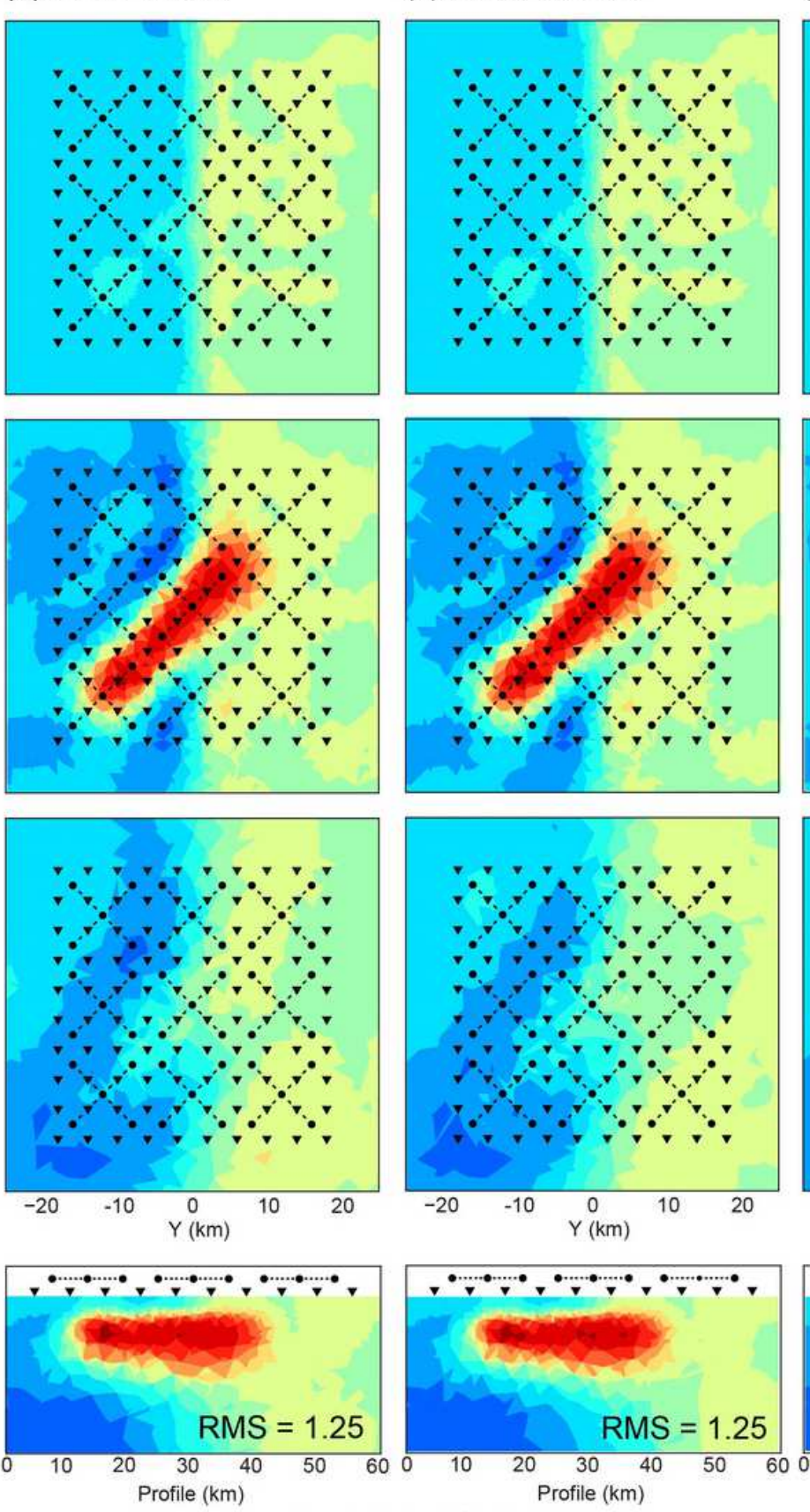

(d) From $1000 \Omega \mathrm{m}$
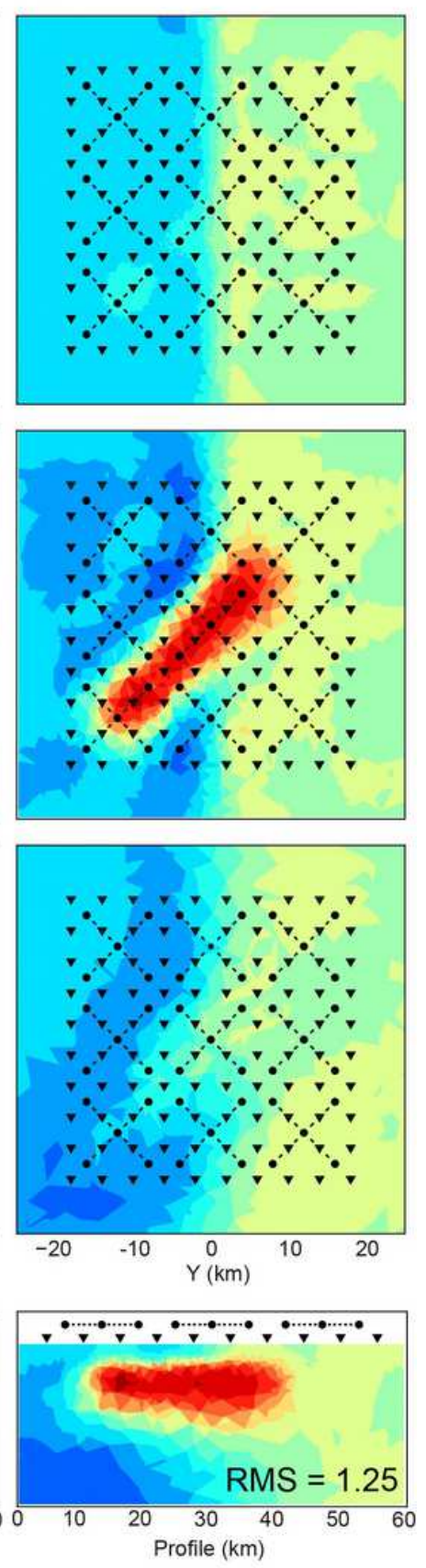

Resistivity $(\Omega \mathrm{m})$

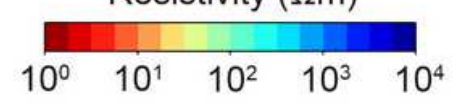

\section{Figure 3}

Electrical resistivity structures obtained by Comb-B. (a) True resistivity structure (OC model). (b)-(c) Resultant resistivity structures obtained by the combined inversion with three different initial models. The lowermost panels indicate the vertical cross-sections of the profile along the major axis of the oblique conductor, which is shown as a white line in the upper-left panel. The inverted triangles indicate the locations of observation stations of PT, VMTF, and HMTF. The broken lines with circular ends indicate the dipoles of the NMT stations. We used the bottom-right point (the station at $x=-18 \mathrm{~km}$ and $y=18 \mathrm{~km}$ ) as the reference station for calculating HMTF and NMTRF. 
(a) $x=10 \mathrm{~km}, y=-10 \mathrm{~km}$

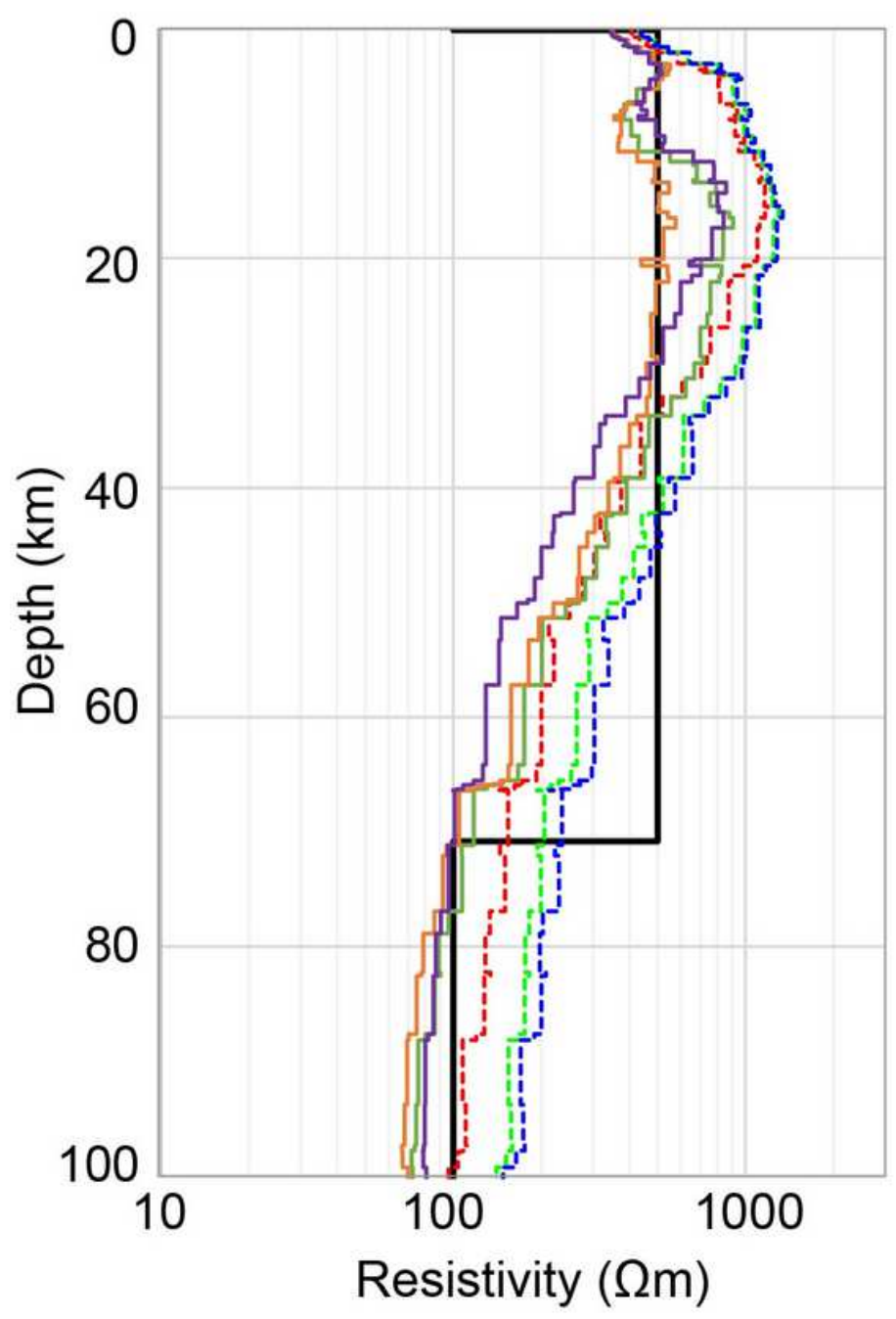

- True model

------ Comb-A (From $10 \Omega \mathrm{m}$ halfspace)

Comb-A (From $100 \Omega m$ halfspace)

------ Comb-A (From $1000 \Omega m$ halfspace) (b) $x=-10 \mathrm{~km}, y=10 \mathrm{~km}$

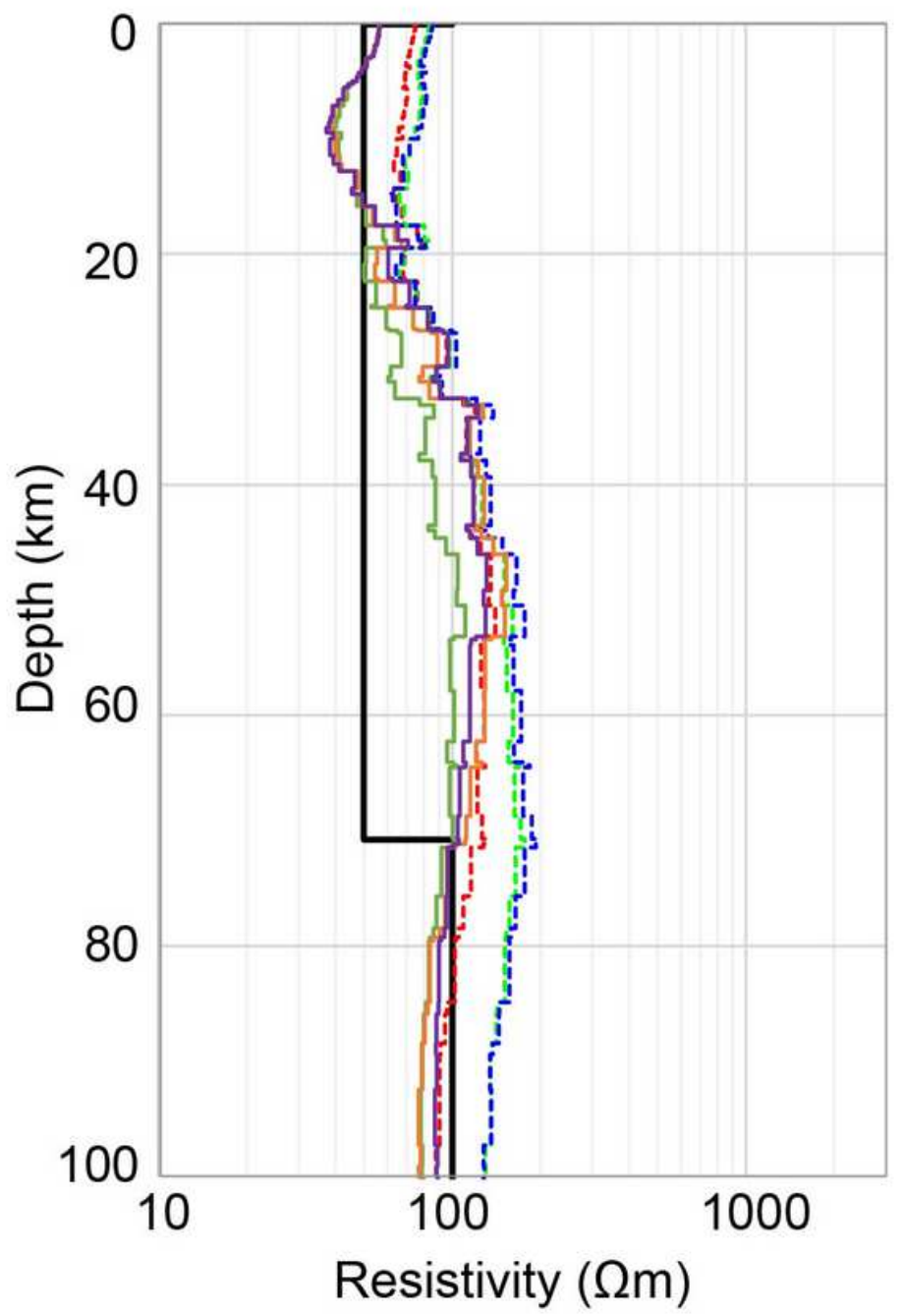

\section{Figure 4}

Electrical resistivity variation with a depth at $(a)(x, y)=(10 \mathrm{~km},-10 \mathrm{~km})$ and $(b)(x, y)=(-10 \mathrm{~km}, 10 \mathrm{~km})$. The thick black lines indicate the resistivity variation of the true resistivity structure. The broken colored and solid-colored lines indicate the resistivity variations of the resistivity structure obtained by Comb-A and Comb-B, respectively. 


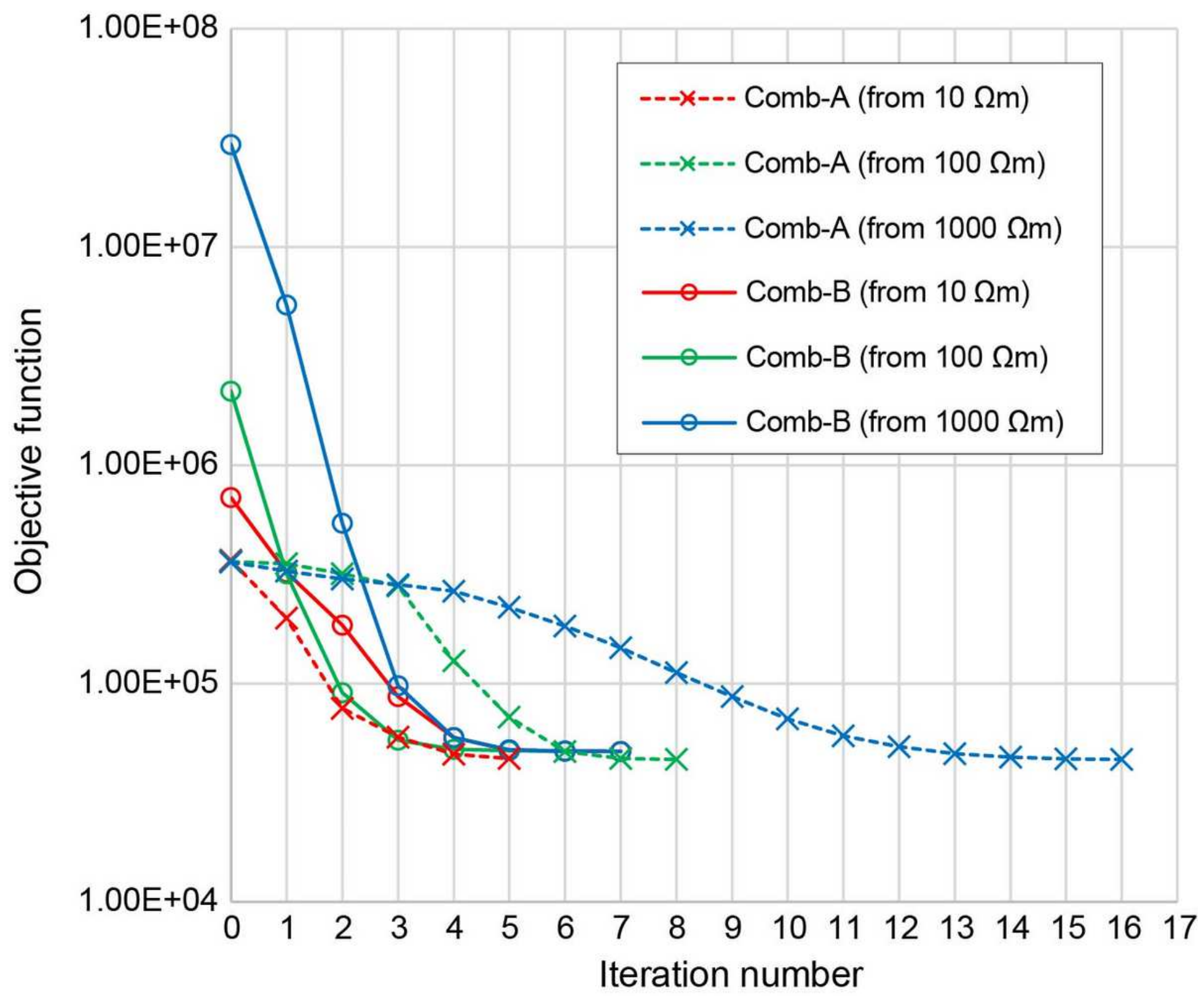

Figure 5

Objective function versus the iteration number for combined inversions with different initial models. The broken and solid lines indicate the objective function changes during Comb-A and Comb-B inversions.

\section{Supplementary Files}

This is a list of supplementary files associated with this preprint. Click to download.

- GraphicalAbstract.jpg

- supportinginformation.docx 